\title{
Melatonin and its agonists: an update
}

\author{
Josephine Arendt and Shantha M. W. Rajaratnam
}

\section{Summary}

The pineal hormone melatonin is able to shift the timing of circadian rhythms, including the sleep-wake cycle, and to promote sleep. Melatonin agonists with similar properties have therapeutic potential for the treatment of circadian rhythm sleep disorders. Depression is specifically targeted by agomelatine, which is also a serotonin-2C (5- $\left.\mathrm{HT}_{2 \mathrm{C}}\right)$ antagonist

\section{Declaration of interest}

S.M.W.R has received research funding and unrestricted educational grants from Vanda Pharmaceuticals (developers of tasimelteon) and Takeda Pharmaceuticals North America (developers of ramelteon). J.A. is a consultant to Alliance Pharmaceuticals, UK (currently developing melatonin formulations). She is a director of Stockgrand Ltd, University of Surrey, UK, a company that sells measurement technology for melatonin and other substances.
Josephine Arendt (pictured) is a chronobiologist and endocrinologist who has studied the physiology and pharmacology of melatonin. Shantha M. W. Rajaratnam is a chronobiologist and sleep researcher who has conducted clinical trials of melatonin and melatonin agonists.

Melatonin (N-acetyl-5-methoxytryptamine) has been credited with many properties since its discovery in 1958. Foremost among these are an ability to provide a biological signal for dawn and dusk, to change the timing of the 'internal clock', which for mammals is located in the suprachiasmatic nuclei of the hypothalamus, and to promote sleepiness or sleep. ${ }^{1,2}$

\section{Endogenous melatonin}

Melatonin is primarily synthesised by the pineal gland with a marked circadian rhythm normally peaking during darkness. Its major function is to act as a 'photoneuroendocrine transducer' - providing information about day length by its profile of secretion. The time of melatonin secretion, rising in the evening and falling during the morning hours, has been referred to as 'biological night.'.,

In humans peak values of melatonin concentration are associated with the lowest point in rhythms of core body temperature, alertness, mental performance and many metabolic functions, and with maximum sleep propensity. ${ }^{3}$ If sleep is timed to occur outside the biological night, its quality and duration are compromised. For these reasons endogenous melatonin is thought to facilitate and reinforce sleep and other aspects of night-time physiology, when it is appropriately timed. ${ }^{4}$

Abnormal rhythms of melatonin have frequently (but inconsistently) been reported in psychiatric disorder, with reports of advanced or delayed phase and a possible decline or increase in amplitude in depression. There appears to be no consensus as to precisely the abnormality present in various types of depression, or indeed if there is one. In most patients with seasonal affective disorder there is probably a delay in melatonin secretion; ${ }^{5}$ for other affective disorders, the pattern remains unclear.

\section{Exogenous melatonin}

Exogenous melatonin has both acute and phase-shifting effects on sleep ${ }^{6}$ and on more strongly endogenous circadian rhythms such as core body temperature. ${ }^{1}$ These effects depend critically on the time of administration. ${ }^{1}$ For example, taken in the afternoon or early evening exogenous melatonin will advance, and taken in the early morning it is reported to delay, the circadian clock. Melatonin treatment during night-time, when endogenous melatonin is secreted, has little effect on sleep but during daytime it induces sleepiness and promotes sleep. There is some evidence that these effects are dose dependent.

Melatonin has obvious therapeutic potential and there has been some success in treating circadian rhythm sleep disorders such as delayed sleep phase syndrome, non-24-h sleep-wake cycle (frequent in people with blindness with no light perception) and, less consistently, the sleep problems of jet lag and shift work. ${ }^{1}$ Limited data suggest that treatment with melatonin itself may be useful in various types of depression ${ }^{7}$ particularly if rhythm abnormalities are present. ${ }^{5}$ It is of interest to note that a number of antidepressant drugs increase peripheral melatonin levels.

\section{Why develop melatonin analogues?}

Melatonin is available over the counter in the USA. However, in most other countries its availability is restricted, and only recently has one particular formulation been approved for prescription use in Europe (www.emea.europa.eu/humandocs/Humans/EPAR/ circadin/circadin.htm). Other formulations are also being developed. Pharmacokinetic properties differ depending on formulation. Melatonin is probably safe in low doses.

The discovery, cloning and characterisation of two principal melatonin membrane receptors $\left(\mathrm{Mel}_{1 \mathrm{a}}\right.$ and $\mathrm{Mel}_{1 \mathrm{~b}}$, now renamed $\mathrm{MT}_{1}$ and $\mathrm{MT}_{2}$ ), found in numerous locations both in the central nervous system (notably within the suprachiasmatic nuclei) and the periphery, has led to much activity designed to synthesise and evaluate indolic and non-indolic analogues specific for either or both of these receptors. ${ }^{8}$ The $\mathrm{MT}_{3}$ receptor is an enzyme, quinone reductase $2,{ }^{8}$ concerned with detoxification mechanisms (not considered here).

\section{Halogenated melatonin}

Probably the first analogues to be synthesised were 6- and 2halogenated melatonins.

Most recently beta-methyl-6-chloromelatonin (LY156735) is under scrutiny as a potential treatment for insomnia and for jet lag. The first trials reported faster adaptation to a simulated time shift with LY156735, ${ }^{9}$ and a randomised placebo-controlled trial showed efficacy of the compound in the treatment of primary insomnia. ${ }^{10}$ 


\section{Agomelatine}

Agomelatine is a potent oral agonist at $\mathrm{MT}_{1}$ and $\mathrm{MT}_{2}$, and an antagonist at serotonin-2C $\left(5-\mathrm{HT}_{2 \mathrm{C}}\right)$, receptors. This compound was initially investigated as a chronobiotic (a substance that shifts rhythms); however, with the discovery of the $5-\mathrm{HT}_{2 \mathrm{c}}$ antagonist activity the emphasis shifted to its anxiolytic and antidepressant effects. In major depressive disorder results from pooled data from three trials ${ }^{11}$ confirmed the efficacy, tolerability and improvement of sleep quality of agomelatine in severe depression. Recently, a randomised trial of agomelatine $(25-50 \mathrm{mg})$ in patients with moderate to severe major depressive disorder $(n=238)$ reported significant improvement in depression severity (Hamilton Rating Scale for Depression, agomelatine-placebo difference of 3.44) with agomelatine. ${ }^{12}$ The efficacy appears similar to, for example, venlafaxine, but with greater improvement in subjective sleep. ${ }^{13}$ The product appears to have no side-effects, including the daytime drowsiness and sexual problems associated with some other antidepressant medications. It is not addictive and there are no withdrawal problems. It does not bind to a broad range of other receptors and thus has a good safety profile, without the problems associated with tricyclics and selective serotonin reuptake inhibitors. Recently it has been reported to prevent relapse into depression. $^{14}$

Since agomelatine has clear chronobiotic activity as well as classic antidepressant activity, the implication is that it is targeting multiple systems, in contrast to the emphasis normally placed on selectivity in receptor targeting. It appears to restore restful sleep and improve daytime alertness by normalising the timing and continuity of sleep in patients with depression. Thus, in addition to major depressive disorder, it is likely to find many applications in sleep disorders.

\section{Ramelteon}

Ramelteon, a selective $\mathrm{MT}_{1} / \mathrm{MT}_{2}$ agonist, is the first in this new class of sleep aids to be approved by the US Food and Drug Administration for long-term use for the treatment of insomnia characterised by difficulty falling asleep. Ramelteon has no important interactions with other receptors including, interestingly, $\mathrm{MT}_{3}$. In addition to sleep-promoting effects, a preclinical study found that ramelteon has chronobiotic properties, indicating potential for development as a treatment for circadian rhythm sleep disorders. ${ }^{15}$

Randomised controlled trials of ramelteon have been reported. ${ }^{16}$ In healthy adults experiencing transient insomnia associated with a novel sleep environment, and in patients with chronic primary insomnia, ramelteon decreased latency to sleep onset and increased total sleep time. These effects were modest overall (latency to persistent sleep 10-19 min; total sleep time 8-22 min), and the clinical significance of such improvements is unclear. It is likely that had patients been selected specifically for sleeptiming problems, greater improvement of sleep might have been seen. The reported side-effects were similar to those of melatonin (sleepiness $5 \%$, fatigue $4 \%$, nausea $3 \%$ ). It is not clear whether or not there is a dose-response relationship at the doses employed. Ramelteon treatment appears to be well tolerated.

\section{Tasimelteon}

Tasimelteon (VEC-162), previously BMS-214778, is a melatonin receptor agonist with high affinity for both the human $\mathrm{MT}_{1}$ and $\mathrm{MT}_{2}$ receptor. Preclinical studies are reported to show that it has similar phase-shifting properties to melatonin, but with less vasoconstrictive effects. ${ }^{17} \mathrm{~A}$ recent report supports the use of tasimelteon in the treatment of circadian rhythm sleep disorders. ${ }^{18}$
Tasimelteon is entering Phase III trials for the treatment of insomnia.

Large numbers of other melatonin receptor agonists and antagonists exist, with varying properties. Thus far, there are no available reports of their use in humans.

\section{Dosage and timing considerations}

It is curious and interesting that doses of agomelatine and ramelteon, the two compounds for which the most information is available, appear to be high compared with the effective dose range for melatonin used as a chronobiotic or soporific. The daily evening dose of agomelatine for depression is at present $25 \mathrm{mg}$, the usual dose of ramelteon appears to be $8 \mathrm{mg}$ daily in the evening, with reports of 4-64 mg being effective for sleep latency, sleep efficiency and total sleep time. In contrast, melatonin is able to entrain the free-running rhythms of most people with blindness at a timed dose of $0.5 \mathrm{mg}$ daily. When given in the early evening, before the endogenous secretion rises, it has soporific activity in healthy adults in the dose range $0.1-10 \mathrm{mg}$, comparable with temazepam (see Arendt ${ }^{1}$ ).

Melatonin must be timed appropriately for maximum efficacy. If melatonin agonists are, like melatonin, most efficacious when given during the 'biological day', the question arises as to whether the compounds are most suitable for the treatment of primary insomnia rather than circadian rhythm sleep disorders. The interest of agomelatine is that it addresses the issue of depression as a primary target. In limited comparisons with available antidepressant medication it appears to have advantages with respect to safety profile, tolerability and normalisation of sleep. However, the National Prescribing Centre website states that, should agomelatine be licensed, 'On the basis of currently available evidence, initial use of agomelatine by prudent clinicians will be limited' (www.npci.org.uk/blog/?p=38).

\section{Comparisons with melatonin}

There are few comparisons regarding the effects of melatonin for any of these analogues. The chronobiotic and thermoregulatory properties of agomelatine were investigated in humans and proved to be very similar to melatonin. The effects of these compounds on sleep also appear to be similar. However, there are insufficient data to decide whether the agonists are preferable to the 'natural' compound.

\section{Conclusions}

Melatonin has proved useful in the treatment of certain sleep disorders. Melatonin agonists provide the clinician with new tools to address insomnia and, in the case of agomelatine, depression; however, their use in circadian rhythm sleep disorders has not been fully explored. Their main advantage is their good safety profile. Whether efficacy is improved compared with the natural compound remains to be determined. Large randomised controlled trials in this area are warranted.

Josephine Arendt, PhD, FRCPath, Centre for Chronobiology, School of Biomedica and Molecular Sciences, University of Surrey, Guildford, UK; Shantha M. W. Rajaratnam, PhD, School of Psychology, Psychiatry and Psychological Medicine, Monash University, Victoria, Australia

Correspondence: Josephine Arendt, Centre for Chronobiology, School of Biomedical and Molecular Sciences, University of Surrey, Guildford, Surrey GU2 7XH, UK. Email: ArendtJo@aol.com

First received 6 Feb 2008, final revision 23 Feb 2008, accepted 29 Apr 2008 


\section{References}

1 Arendt J. Melatonin: characteristics, concerns, and prospects. J Biol Rhythms 2005; 20: 291-303.

2 Wehr TA, Duncan WC Jr, Sher L, Aeschbach D, Schwartz PJ, Turner EH, Postolache TT, Rosenthal NE. A circadian signal of change of season in patients with seasonal affective disorder. Arch Gen Psychiatry 2001; 58: 1108-14.

3 Rajaratnam SMW, Arendt J. Health in the 24-hour society. Lancet 2001; 358 999-1005.

4 Dijk DJ, Lockley Sw. Integration of human sleep-wake regulation and circadian rhythmicity (Invited Review). J Appl Physiol 2002; 92: 852-62.

5 Lewy AJ, Lefler BJ, Emens JS, Bauer VK. The circadian basis of winter depression. Proc Natl Acad Sci USA 2006; 103: 7414-9.

6 Rajaratnam SM, Middleton B, Stone BM, Arendt J, Dijk DJ. Melatonin advances the circadian timing of EEG sleep and directly facilitates sleep without altering its duration in extended sleep opportunities in humans. J Physiol 2004; 561 (Pt 1): 339-51.

7 Serfaty MA, Osborne D, Buszewicz MJ, Raven PW. The effects of exogenous melatonin in major depression. Chronobiol Int 2003; 20: 1191-3.

8 Dubocovich ML. Melatonin receptors: role on sleep and circadian rhythm regulation. Sleep Med 2007; 8 (suppl 3): 34-42.

9 Nickelsen T, Samel A, Vejvoda M, Wenzel J, Smith B, Gerzer R. Chronobiotic effects of the melatonin agonist LY 156735 following a simulated $9 \mathrm{~h}$ time shift: results of a placebo-controlled trial. Chronobiol Int 2002; 19: 915-36.

10 Zemlan FP, Mulchahey JJ, Scharf MB, Mayleben DW, Rosenberg R, Lankford A. The efficacy and safety of the melatonin agonist beta-methyl-6- chloromelatonin in primary insomnia: a randomized, placebo-controlled, crossover clinical trial. J Clin Psychiatry 2005; 66: 384-90.

11 Montgomery SA. Major depressive disorders: clinical efficacy and tolerability of agomelatine, a new melatonergic agonist. Eur Neuropsychopharmacol 2006; 16: s633-8

12 Olie JP, Kasper S. Efficacy of agomelatine, a MT1/MT2 receptor agonist with 5-HT2C antagonistic properties, in major depressive disorder. Int $J$ Neuropsychopharmacol 2007; 10: 661-73.

13 Lemoine $P$, Guilleminault $C$, Alvarez E. Improvement in subjective sleep in major depressive disorder with a novel antidepressant, agomelatine: randomized, double-blind comparison with venlafaxine. J Clin Psychiatry 2007; 68: 1723-32.

14 Goodwin GM. Effective management of depressed mood with agomelatine, a melatonergic antidepressant Eur Psychiatry 2007; 22 (suppl 1): s91.

15 Hirai K, Kita M, Ohta H, Nishikawa H, Fujiwara $\mathrm{Y}$, Ohkawa S, Miyamoto M. Ramelteon (TAK-375) accelerates reentrainment of circadian rhythm after a phase advance of the light-dark cycle in rats. J Biol Rhythms 2005; 20: 27-37.

16 Borja NL, Daniel KL. Ramelteon for the treatment of insomnia. Clin Ther 2006; 28: $1540-55$.

17 Vachharajani NN, Yeleswaram K, Boulton DW. Preclinical pharmacokinetics and metabolism of BMS-214778, a novel melatonin receptor agonist. J Pharm Sci 2003; 92: 760-72.

18 Rajaratnam SM, Polymeropoulos MH, Fisher DM, Roth T, Scott C, Birznieks G, Klerman EB. Randomised controlled trials of the melatonin agonist tasimelteon (VEC-162) for transient insomnia after sleep time shift. Lancet, in press. 